\title{
Analytical solution to the Schrödinger equation of a laser-driven correlated two-particle system
}

\author{
Uwe Schwengelbeck \\ Departamento de Física Aplicada, Universidad de Salamanca, \\ E-37008 Salamanca, Spain
}

March 29, 2021

\begin{abstract}
The time-dependent quantum system of two laser-driven electrons in a harmonic oscillator potential,is analysed, taking into account the repulsive Coulomb interaction between both particles. The Schrödinger equation of the two-particle system is shown to be analytically soluble in case of arbitrary laser frequencies and individual oscillator frequencies, defining the system. Quantum information processing could be a possible field of application.
\end{abstract}

PACS numbers: 42.50.Ge, 03.65.Ge, 33.80†

There is an inherent interest in analytical and non-perturbative solutions of time-dependent quantum problems, all the more considering quantum systems with more than one particle. One of the few examples of such kind is the system of two electrons in an electromagnetic field [1], where an analytical solution of the Schrödinger equation has been given recently [2]. The aim of this work is to demonstrate that exact wavefunctions are available for the time-dependent problem of two correlated charged particles in a harmonic oscillator potential, subject to a laser field (see also Ref. [3]). The given analytical solutions [3] are valid for arbitrary driving laser frequencies and individual oscillator frequencies, defining the system. A possible field of application might be quantum information processing (cf., e.g., Ref. 四). Another application of the timedependent solution to the two-particle Schrödinger equation could be the test of costly numerical algorithms to simulate the dynamics of helium in intense laser fields - still one of the topical problems in theoretical atomic physics these days. The latter system has, apart from the Coulomb interaction between the electrons and the nucleus, a similar structure as the system considered here, containing a harmonic potential.

The wavefunction $\Psi$ of two laser-driven particles of equal mass $\mu$ and charge $q$ in a harmonic potential, associated with the oscillator frequency $\Omega$, obeys the Schrödinger equation

$$
i \hbar \frac{\partial}{\partial t} \Psi\left(\mathbf{r}_{1}, \mathbf{r}_{2}, t\right)=\hat{H}\left(\mathbf{r}_{1}, \mathbf{r}_{2}, t\right) \Psi\left(\mathbf{r}_{1}, \mathbf{r}_{2}, t\right),
$$


where the Hamiltonian in length gauge [5] reads

$$
\hat{H}\left(\mathbf{r}_{1}, \mathbf{r}_{2}, t\right)=\sum_{i=1}^{2}\left[\frac{\hat{\mathbf{p}}_{i}^{2}}{2 \mu}+\frac{\mu}{2} \Omega^{2} \mathbf{r}_{i}{ }^{2}-q \mathbf{r}_{i} \cdot \hat{\mathbf{e}} E_{0} \sin (\omega t+\delta)\right]+V\left(\mathbf{r}_{1}, \mathbf{r}_{2}\right),
$$

$\omega$ and $E_{0}$ are the frequency and the amplitude of a monochromatic laser field, respectively, ê denotes a unit polarization vector, and

$$
V\left(\mathbf{r}_{1}, \mathbf{r}_{2}\right)=\frac{q^{2}}{\left|\mathbf{r}_{1}-\mathbf{r}_{2}\right|}
$$

describes the represents Coulomb interaction between both particles.

On using center of mass and relative coordinates,

$$
\mathbf{R}=\frac{1}{2}\left(\mathbf{r}_{1}+\mathbf{r}_{2}\right) \quad \text { and } \quad \mathbf{r}=\mathbf{r}_{1}-\mathbf{r}_{2},
$$

the Hamiltonian (2) decouples,

$$
\hat{H}=\frac{\hat{\mathbf{P}}^{2}}{4 \mu}+\frac{\hat{\mathbf{p}}^{2}}{\mu}+\mu \Omega^{2} \mathbf{R}^{2}+\frac{\mu}{4} \Omega^{2} \mathbf{r}^{2}-2 q \mathbf{R} \cdot \hat{\mathbf{e}} E_{0} \sin (\omega t+\delta)+\frac{q^{2}}{|\mathbf{r}|},
$$

where

$$
\hat{\mathbf{P}}=\hat{\mathbf{p}}_{1}+\hat{\mathbf{p}}_{2}=-i \hbar \nabla_{\mathbf{R}}, \quad \text { and } \quad \hat{\mathbf{p}}=\frac{1}{2}\left(\hat{\mathbf{p}}_{1}-\hat{\mathbf{p}}_{2}\right)=-i \hbar \nabla_{\mathbf{r}},
$$

and the Schrödinger equation (1) admits solutions of the factorized form

$$
\Psi=\psi(\mathbf{R}, t) \phi(\mathbf{r}, t) \chi(1,2),
$$

where $\chi(1,2)$ is the corresponding spin state of the two-particle system. The respective differential equations for the wavefunctions $\psi(\mathbf{R}, t)$ and $\phi(\mathbf{r}, t)=$ $\phi(\mathbf{r}) \exp (-i \epsilon t / \hbar)$ in $(7)$ then read

$$
i \hbar \frac{\partial}{\partial t} \psi(\mathbf{R}, t)=\left[\frac{\hat{\mathbf{P}}^{2}}{4 \mu}+\mu \Omega^{2} \mathbf{R}^{2}-2 q \mathbf{R} \cdot \hat{\mathbf{e}} E_{0} \sin (\omega t+\delta)\right] \psi(\mathbf{R}, t)
$$

and

$$
\epsilon \phi(\mathbf{r})=\left(\frac{\hat{\mathbf{p}}^{2}}{\mu}+\frac{\mu}{4} \Omega^{2} \mathbf{r}^{2}+\frac{q^{2}}{|\mathbf{r}|}\right) \phi(\mathbf{r}) .
$$

For both of the above equations, (8) and (9), analytical solutions are available [3].

Equation (8), associated with the center of mass motion of the two particles, has essentially the form of the Schrödinger equation of a driven harmonic oscillator. Given an initial wavefunction $\psi(\mathbf{R}, 0)$, the solution of equation (8) can be obtained by means of a path integral [6]:

$$
\psi(\mathbf{R}, t)=\int_{-\infty}^{\infty} K\left(\mathbf{R}, t ; \mathbf{R}^{\prime}, 0\right) \psi\left(\mathbf{R}^{\prime}, 0\right) d \mathbf{R}^{\prime},
$$


where the propagator, corresponding to equation (8), reads

$$
K\left(\mathbf{R}, t ; \mathbf{R}^{\prime}, 0\right)=\sqrt{\frac{\mu \Omega}{i \pi \hbar \sin (\Omega t)}} e^{\frac{i}{\hbar} S\left(\mathbf{R}, t ; \mathbf{R}^{\prime}, 0\right)},
$$

containing the action function

$$
\begin{aligned}
S= & \frac{\mu \Omega}{\sin (\Omega t)}\left[\left(\mathbf{R}^{\prime 2}+\mathbf{R}^{2}\right) \cos \Omega t-2 \mathbf{R}^{\prime} \cdot \mathbf{R}\right. \\
& +\frac{2 q E_{0}}{\mu \Omega} \int_{0}^{t} \mathbf{R} \cdot \hat{\mathbf{e}} \sin (\omega \tau+\delta) \sin \Omega \tau d \tau \\
& +\frac{2 q E_{0}}{\mu \Omega} \int_{0}^{t} \mathbf{R}^{\prime} \cdot \hat{\mathbf{e}} \sin (\omega \tau+\delta) \sin [\Omega(t-\tau)] d \tau \\
- & \left.\frac{2 q^{2} E_{0}^{2}}{\mu^{2} \Omega^{2}} \int_{0}^{t} \int_{0}^{\tau} \sin (\omega \tau+\delta) \sin (\omega s+\delta) \sin [\Omega(t-\tau)] \sin \Omega s d s d \tau\right]
\end{aligned}
$$

As an example we shall consider here a laser field with linear polarization along the $z$-direction and phase $\delta=0$. On starting with the unperturbed oscillator ground state

$$
\psi(\mathbf{R}, 0)=\left(\frac{2 \mu \Omega}{\pi \hbar}\right)^{3 / 4} e^{-\frac{\mu}{\hbar} \Omega \mathbf{R}^{2}},
$$

the center of mass wavefunction at time $t$, given by integral (10), reads $(\mathbf{R}=$ $(X, Y, Z))$

$$
\begin{aligned}
\psi(\mathbf{R}, t)= & \left(\frac{2 \mu \Omega}{\pi \hbar}\right)^{3 / 4} e^{-\frac{3}{2} i \Omega t} e^{-\frac{\mu}{\hbar} \Omega\left(X^{2}+Y^{2}\right)} e^{i \frac{\mu}{\hbar} \Omega Z^{2} \cot \Omega t} \\
& \times \exp \left(-\frac{\mu \Omega\left(q E_{0} \frac{\sin \omega t-\frac{\omega}{\Omega} \sin \Omega t}{\mu\left(\Omega^{2}-\omega^{2}\right)}-Z\right)^{2}}{\hbar \sin ^{2} \Omega t(1-i \cot \Omega t)}\right) \\
& \times \exp \left(2 i q E_{0} Z \frac{\omega \cos \omega t-\Omega \sin \omega t \cot \Omega t}{\hbar\left(\Omega^{2}-\omega^{2}\right)}\right) \\
& \times \exp \left(-i q^{2} E_{0}^{2} \frac{\sin \omega t \cos \omega t\left(\omega+\frac{\Omega^{2}}{\omega}\right)-2 \Omega \cot \Omega t \sin ^{2} \omega t}{2 \mu \hbar\left(\Omega^{2}-\omega^{2}\right)^{2}}\right) \\
& \times \exp \left(\frac{i q^{2} E_{0}^{2} t}{2 \mu \hbar\left(\Omega^{2}-\omega^{2}\right)}\right),
\end{aligned}
$$

which can be verified directly by substitution into equation (8). It may be noted that for the case of a circularly polarized laser field, the wavefunction can be obtained in a similar way as in the above case with a linearly polarized laser field.

The remaning problem (9), concerning the relative motion of both electrons, is analytically soluble for an infinite denumerable set of oscillator frequencies 
$\Omega[7,8,9$. On using spherical polar coordinates, the relative coordinate wavefunction reads

$$
\phi(\mathbf{r})=\frac{u(r)}{r} Y_{l m}(\theta, \phi)
$$

where $Y_{l m}$ are the spherical harmonics, and the radial part, given by the ansatz

$$
u(r)=r^{l+1} e^{-\frac{\mu}{4 \hbar} \Omega r^{2}} \sum_{\nu=0}^{\infty} a_{\nu} r^{\nu}
$$

is determined by

$$
\epsilon u(r)=\left[-\frac{\hbar^{2}}{\mu} \frac{d^{2}}{d r^{2}}+\frac{\mu}{4} \Omega^{2} r^{2}+\frac{q^{2}}{r}+\frac{\hbar^{2} l(l+1)}{\mu r^{2}}\right] u(r) .
$$

In closed form solutions of the radial equation (17), only a finite number of coefficients $a_{\nu} \neq 0$ contributes to the power series expansion in equation (16), which is satisfied in particular cases of harmonic oscillator frequencies $\Omega$. As an example, an account of closed form solutions shall be given here for oscillator frequencies, related to the angular momentum quantum numbers $l=0,1, \ldots, \infty$ by (cf. Ref. [8])

$$
\Omega=\frac{q^{4} \mu}{2 \hbar^{3}(l+1)} .
$$

The eigenfunction (15) for the relative motion of the two particles is then given by

$$
\phi(\mathbf{r})=r^{l} e^{-\frac{\mu}{4 \hbar} \Omega r^{2}}\left(1+\frac{\hbar}{q^{2}} \Omega r\right) Y_{l m}(\theta, \phi),
$$

corresponding to an energy $\epsilon=\frac{\hbar}{2}\left(3 \Omega+\frac{q^{4} \mu}{\hbar^{3}}\right)$. For a numerical treatment of equation (9) yielding solutions valid for arbitrary values of oscillator frequency $\Omega$, the reader may be refered to Refs. [10, 11].

It is noted that the center of mass wavefunction (14), comprising the dynamical evolution of the system subject to the laser field, remains symmetric against particle exchange. At the same time, the total wavefunction $\Psi=$ $\psi(\mathbf{R}, t) \phi(\mathbf{r}, t) \chi(1,2)$ has, according to the Pauli principle, to be antisymmetric against particle exchange. Thus, in case of antisymmetric solutions of equation (9) with $\phi(\mathbf{r})=-\phi(-\mathbf{r})$, the spin state $\chi(1,2)$ of the system has to be symmetric against particle exchange, leading to triplet states. On the other hand, symmetric functions $\phi(\mathbf{x})$ must be accompanied by an antisymmetric spin state $\chi(1,2)=-\chi(2,1)$, describing singlet states of the system. The laser interaction does not change the parity of the system wavefunction. In case of a vanishing harmonic oscillator potential, i.e. $\Omega=0$ in Hamiltonian (2), the system reduces to that of two "free" particles in a laser field, discussed in Ref. [2]. The relative motion of both particles is then represented by repulsive Coulomb wavefunctions, satisfying either ingoing or outgoing boundary conditions. These solutions do not permit a motion of both particles with equal kinetic energy and wave vectors in the same direction [2]. In contrast to that case, the time-dependent 
center of mass wavefunction (8) and the stationary relative coordinate wavefunction (9) of the system, based on the repulsive Coulomb and the harmonic potential term, describe two laser-driven particles of which both contributions are always pointed towards the same direction.

In conclusion, the time-dependent problem of two laser-driven correlated particles in a harmonic potential has been shown to be analytically soluble. The Hamiltonian of the Schrödinger equation of the system can be decoupled into that of a driven harmonic oscillator for the center of mass motion and a Hamiltonian for the relative motion, comprising the repulsive Coulomb interaction between both particles. For both the center of mass part and the relative part of the two-particle problem, analytical solutions are available. Besides applications concerning laser interaction with ions in a trap, and nuclear systems, the time-dependent wavefunctions may be utilized to check numerical algorithms to determine the dynamical evolution of the wavefunction of two-electron systems, e.g. to simulate the dynamics of the helium atom in laser fields. Another possible field of application might be quantum information processing.

\section{Acknowledgments}

It gives me great pleasure to thank Luis Plaja, Luis Roso, Javier Rodríguez Vázquez de Aldana and Roberto Numico for the stimulating exchange and useful discussions.

\section{References}

[1] J. Bergou, S. Varró, M.V. Fedorov, J. Phys. A 14 (1981) 2305

[2] F.H.M. Faisal, Phys. Lett. A 187 (1994) 180

[3] U. Schwengelbeck, Phys. Lett. A 253 (1999) 168

[4] J. Mompart, R. Corbalan, L. Roso, Phys. Rev. Lett. 88 (2002) 023603

[5] N.B. Delone, V.P. Krainov, Multiphoton Processes in Atoms (SpringerVerlag, Berlin, 1994)

[6] R.P. Feynman, A.R. Hibbs, Quantum Mechanics and Path Integrals (McGraw-Hill, New York, 1965)

[7] S. Kais, D.R. Hershbach, R.D. Levine, J. Chem. Phys. 91 (1989) 7791

[8] M. Taut, Phys. Rev. A 48 (1993) 3561

[9] M. Taut, A. Ernst, H. Eschrig, J. Phys. B 31 (1998) 2689

[10] P.M. Laufer, J.B. Krieger, Phys. Rev. A 33 (1986) 1480

[11] U. Merkt, J. Huser, M. Wagner, Phys. Rev. B 43 (1991) 7320 